\title{
Cystic Fibrosis Mucus Model to Design More Efficient Drug Therapies
}

Cosmin Butnarasu *a, Giulia Caron a, Daniela Peneda Pacheco b,c, Paola Petrini b, Sonja Visentin *a

a University of Torino, Department of Molecular Biotechnology and Health Science, Via Quarello 15, 10135 Torino, Italy

${ }^{b}$ Department of Chemistry, Materials and Chemical Engineering, Giulio Natta-Politecnico di Milano, Piazza Leonardo da Vinci 32, 20133 Milano, Italy

c Bac3Gel Lda, TagusPark - Edificio Inovacao II, 2740-122 Porto Salvo, Portugal.

*Corresponding author: sonja.visentin@unito.it, cosminstefan.butnarasu@unito.it,

\section{Supporting Material}

\section{Tables}

Table S1. Analytical conditions of the investigated compounds on LC-MS and LC-UV.

Table S2. Molecular descriptors. Principal Component Analysis was computed using all descriptors except for $P_{a p p}$ and Effect of mucus on $P_{a p p}$.

Table S3. Comparison of the permeability data for some of the tested compounds with what previously reported by Chen et al. ${ }^{1}$

\section{Figures}

Figure S1: Structures of the tested drugs.

Figure S2: Correlation of the Papp obtained in the PAMPA system with the permeability reported by Chen et al. ${ }^{1}$

Figure S3. Correlation matrix.

Figure S4. Principal component analysis of the dataset.

Figure S5. Selection of components based on parallel analysis. By retaining only those PCs with eigenvalues greater than the $95^{\text {th }}$ percentile of the simulated eigenvalues, it is ensured that the variance explained by these PCs likely represents "real" variance and not variance due to noise.

Figure S6. Comparison of the permeability recorded on PAMPA without (blue) and with mucus (red) for the tested compounds.

Figure. S7. The ESI-MS spectrum of the naproxen-calcium complex. 
Table S1. Analytical conditions of the investigated compounds on LC-MS and LC-UV.

\begin{tabular}{|c|c|c|c|c|c|c|c|}
\hline$\#$ & Compounds & $\begin{array}{l}\text { Mobile } \\
\text { phases }\end{array}$ & Polarity & $\begin{array}{c}\text { Parent } \\
\text { ion }\end{array}$ & $\begin{array}{l}\text { Fragment } \\
\text { ion }\end{array}$ & CE (V) & $\begin{array}{c}\text { UV detection } \\
(\mathrm{nm})\end{array}$ \\
\hline 1 & Propranolol & & + & 259.9 & 116.1 & -11.5 & \\
\hline 2 & Baricitinib & & + & 371.7 & 186.2 & -34.5 & \\
\hline 3 & Camostat & & + & 399 & 296.1 & -18 & \\
\hline 4 & Dexamethasone & & + & 372.8 & 237.1 & -20 & \\
\hline 6 & Favipiravir & & - & 155.5 & 112.7 & 13 & \\
\hline 7 & Indinavir & & + & 614.2 & 421.1 & -26.5 & \\
\hline 8 & Oseltamivir & & + & 313 & 166.2 & -15 & \\
\hline 9 & Remdesivir & & + & 603.1 & 200 & -41.5 & \\
\hline 10 & Saquinavir & & + & 671.3 & 570.2 & -25 & \\
\hline 11 & Umifenovir & & + & 479.2 & 433.9 & -15 & \\
\hline 12 & Zanamivir & & + & 333.2 & 273.9 & -15.5 & \\
\hline 13 & Levofloxacin & & + & 362 & 317.9 & -16.5 & \\
\hline 14 & CFTR $_{\text {inh }}-172$ & & - & 408.2 & 363.9 & 7 & \\
\hline 15 & Verapamil & & + & 455.4 & 164.9 & -21 & \\
\hline 17 & Amlodipine & & + & 409.2 & 237.7 & -14.5 & \\
\hline 18 & Amitriptyline & & + & 278.2 & 90.8 & -19 & \\
\hline 19 & Procaine & & + & 237.2 & 99.8 & -13 & \\
\hline 20 & Caffeine & & + & 195.1 & 137.8 & -15.5 & \\
\hline 22 & Piroxicam & H2O FA & + & 332 & 94.8 & -17.5 & \\
\hline 23 & Diclofenac & $0.1 \%-\mathrm{ACN}$ & + & 296 & 213.9 & -35 & \\
\hline 24 & Ampicillin & & + & 350 & 105.8 & -21 & \\
\hline 25 & Tetracycline & & + & 445.2 & 409.8 & -14.5 & \\
\hline 26 & Ritonavir & & + & 721 & 296 & -20 & \\
\hline 28 & Ceftazidime & & + & 547.4 & 79.8 & -9.5 & \\
\hline 29 & Ciprofloxacin & & + & 331.9 & 230.8 & -38.5 & \\
\hline 30 & Rifampicin & & + & 823.6 & 791 & -13 & \\
\hline 31 & Salbutamol & & + & 240 & 147.9 & -18.5 & \\
\hline 32 & Aztreonam & & - & 433.9 & 95.7 & 16 & \\
\hline 34 & Lumacaftor & & + & 453.3 & 413.1 & -18.5 & \\
\hline 35 & Valsartan & & - & 434.2 & 349.7 & 15 & \\
\hline 36 & Lidocaine & & + & 235.1 & 85.6 & -14 & \\
\hline 37 & TUDCA & & - & 498.7 & 123.5 & 16.5 & \\
\hline 38 & GUDCA & & - & 448.7 & 74 & 12 & \\
\hline 39 & Tobramycin & & + & 468 & 324 & -13 & \\
\hline 41 & Acetaminophen & & + & 152 & 109.8 & -20 & \\
\hline 42 & Quinine & & + & 325 & 307 & -18 & \\
\hline 44 & Ketoprofen & & + & 255.1 & 104.7 & -22 & \\
\hline 45 & Antipyrine & & + & 189 & 76.8 & -32.5 & \\
\hline 21 & Naproxen & Ammonium & - & 229 & 169.4 & 13 & \\
\hline 27 & Indomethacin & acetate 5 & - & 356.2 & 312 & 6 & \\
\hline 33 & Cefuroxime & $\mathrm{mM}$ pH 6.6 & - & 423.3 & 206.9 & 9.5 & \\
\hline 40 & Ibuprofen & $-\mathrm{ACN}$ & - & 205 & 160.5 & 5.5 & \\
\hline 41 & Ebselen & & & & & & 263 \\
\hline
\end{tabular}


42 3-aminophenol Ammonium

43 Benzoic acid

acetate 5

$\mathrm{mM}$ pH 6.6 
Table S2. Molecular descriptors. Principal Component Analysis was computed using all descriptors except for $P_{\text {app }}$ and Effect of mucus on $P_{\text {app. }}$.

\begin{tabular}{|c|c|c|}
\hline$\#$ & Molecular descriptor & Origin \\
\hline 1 & Charge at pH 7.4 & MarvinSketch \\
\hline 2 & $\mathrm{P}_{\text {app }}$ (PAMPA) & Experimental \\
\hline 3 & Effect of mucus on $\mathrm{P}_{\mathrm{app}}$ & \\
\hline 4 & Total Molweight & DataWarrior \\
\hline 5 & cLogP & \\
\hline 6 & cLogS & \\
\hline 7 & H-Acceptors & \\
\hline 8 & H-Donors & \\
\hline 9 & Total Surface Area & \\
\hline 10 & Relative PSA & \\
\hline 11 & Total Polar Surface Area & \\
\hline 12 & Druglikeness & \\
\hline 13 & Shape Index & \\
\hline 14 & Molecular Flexibility & \\
\hline 15 & Molecular Complexity & \\
\hline 16 & Non-H Atoms & \\
\hline 17 & Non-C/H Atoms & \\
\hline 18 & Electronegative Atoms & \\
\hline 19 & Stereo Centers & \\
\hline 20 & Rotatable Bonds & \\
\hline 21 & Rings Closures & \\
\hline 22 & Aromatic Atoms & \\
\hline 23 & sp3-Atoms & \\
\hline 24 & Symmetric Atoms & \\
\hline 25 & Small Rings & \\
\hline 26 & Aromatic Rings & \\
\hline 27 & Amides & \\
\hline 28 & Amines & \\
\hline 29 & Alkyl-Amines & \\
\hline 30 & Aromatic Amines & \\
\hline 31 & Aromatic Nitrogens & \\
\hline 32 & Basic Nitrogens & \\
\hline 33 & Acidic Oxygens & \\
\hline
\end{tabular}


Table S3. Comparison of the permeability data for some of the tested compounds with what reported in the literature.

\begin{tabular}{|c|c|c|c|c|}
\hline Compound & \multicolumn{2}{|c|}{$\begin{array}{c}\text { Papp PAMPA } \\
( \pm \text { SD }) \times 10^{-6}[\mathrm{~cm} / \mathrm{s}]\end{array}$} & \multicolumn{2}{|c|}{$\begin{array}{c}\text { Papp }_{\text {PAMPA literature }} \\
\text { reference }( \pm S D) \times 10^{-6}[\mathrm{~cm} / \mathrm{s}]^{1}\end{array}$} \\
\hline Propranolol & 12.56 & $( \pm 2.9)$ & 10.32 & $( \pm 2.4)^{1,2}$ \\
\hline Naproxen & 9.57 & $( \pm 3.9)$ & 6.03 & $( \pm 0.6)$ \\
\hline Caffeine & 12.70 & $( \pm 3.5)$ & 9.89 & $( \pm 1.5)$ \\
\hline Ibuprofen & 9.09 & $( \pm 1.4)$ & 10.73 & $( \pm 1.6)$ \\
\hline Verapamil & 8.57 & $( \pm 2.0)$ & 8.75 & $( \pm 0.5)$ \\
\hline Diclofenac & 4.03 & $( \pm 0.2)$ & 6.95 & $( \pm 0.3)$ \\
\hline Antipyrine & 5.75 & $( \pm 1.7)$ & 7.51 & $( \pm 1.4)$ \\
\hline Ketoprofen & 4.04 & $( \pm 0.7)$ & 4.13 & $( \pm 0.4)$ \\
\hline Piroxicam & 3.15 & $( \pm 0.8)$ & 4.96 & $( \pm 0.7)$ \\
\hline
\end{tabular}




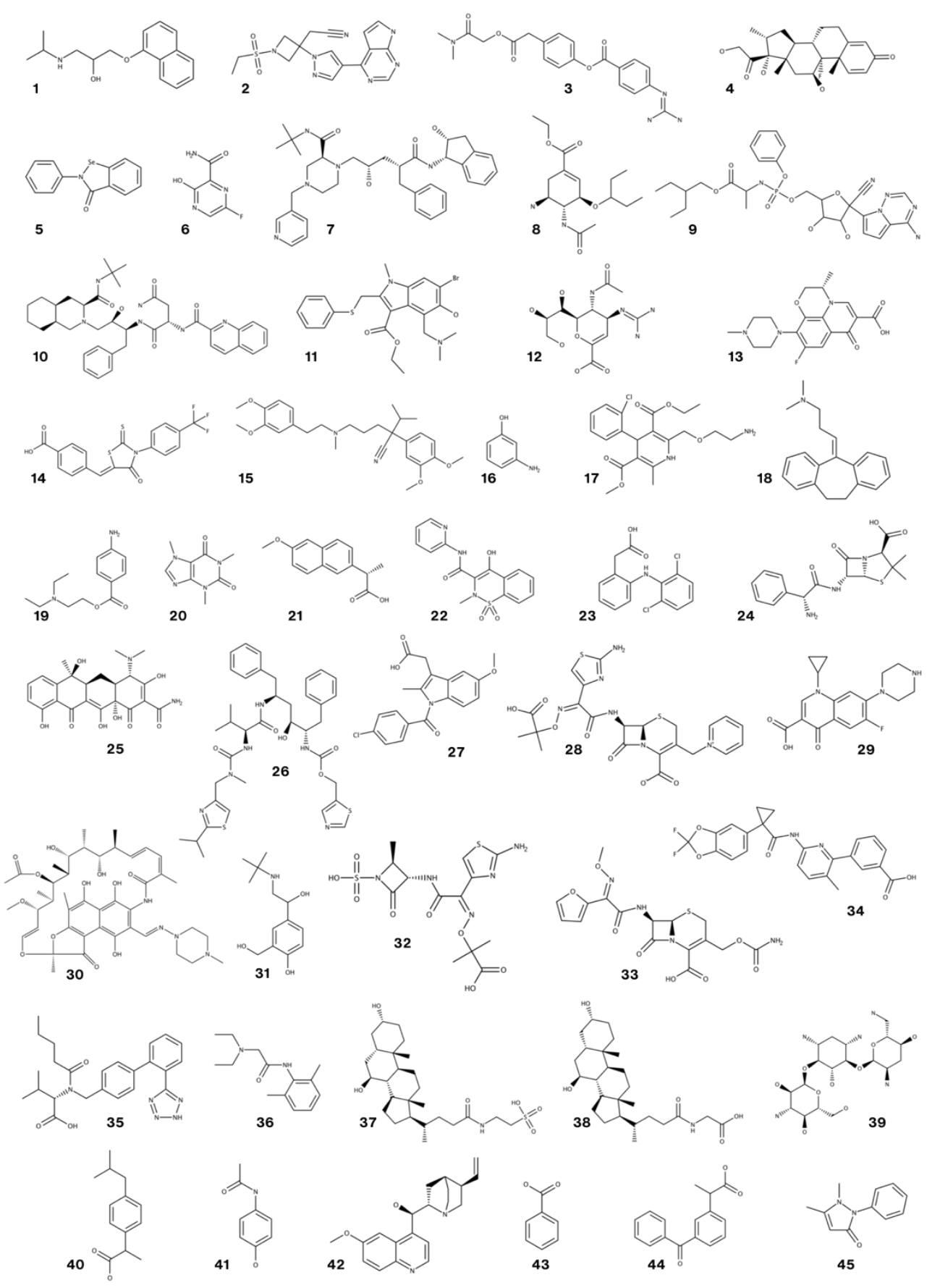

Figure S1. Structures of the tested drugs. (1) propranolol, (2) baricitinib, (3) camostat, (4) dexamethasone, (5) ebselen, (6) favipiravir, (7) indinavir, (8) oseltamivir, (9) remdesivir, (10) saquinavir, (11) umifenovir, (12) zanamivir, (13) levofloxacin, (14) CFTRinh-172, (15) verapamil, (16) 3-aminophenol, (17) amlodipine, (18) amitriptyline, (19) procaine, (20) caffein, (21) naproxen, (22) piroxicam, (23) diclofenac, (24) a mpicillin, (25) tetracycline, (26) ritonavir, (27) indomethacin, (28) ceftazidime, (29) ciprofloxacin, (30) rifampicin, (31) salbutamol, (32) aztreonam, (33) cefuroxime, (34) lumacaftor, (35) valsartan, (36) lidocaine, (37) tauroursodeoxycholic acid, (38) glycoursodeoxycholic acid, (39) tobramycin, (40) ibuprofen, (41) acetaminophen, (42) quinine, (43) benzoic acid, (44) ketoprofen, (45) antipyrine. 


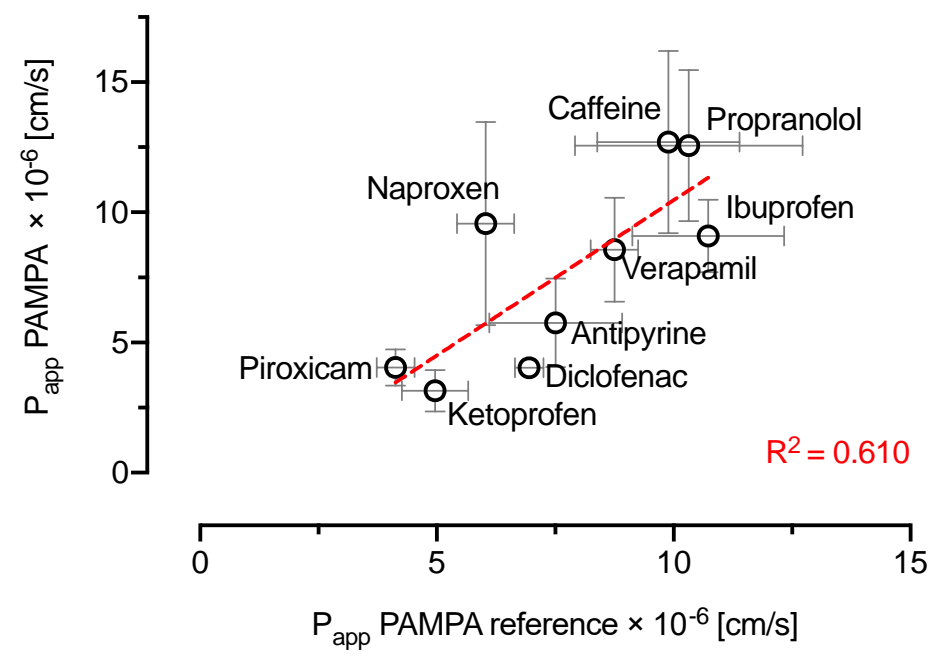

Figure S2. Comparison between PAMPA permeability obtained with the modified setup and PAMPA permeability reported by Chen et al., using the same artificial membrane. Data used in this plot are listed in Table S3.

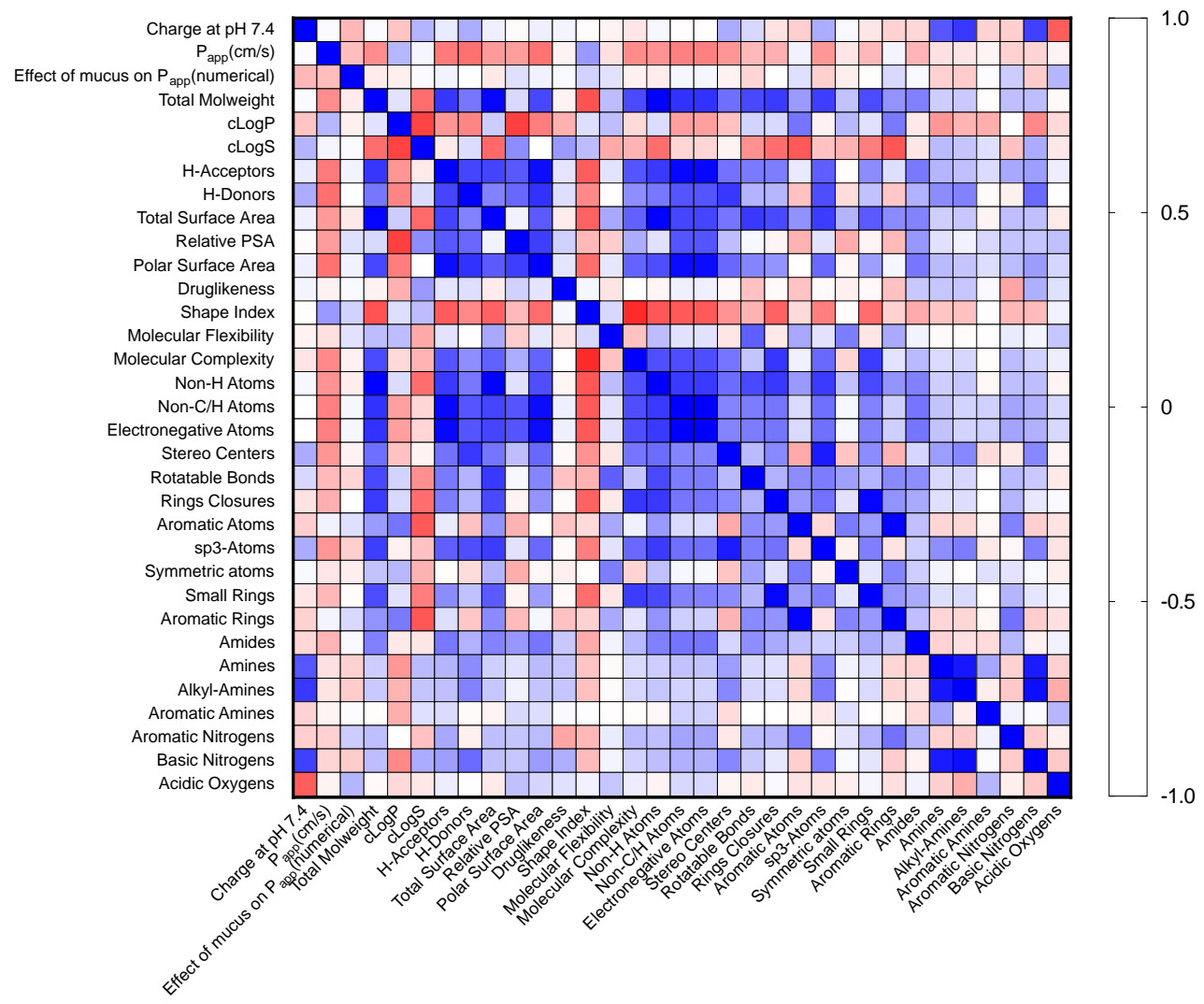

Figure S3. Correlation matrix 
In addition to the discrimination of the high and low permeable compounds based on the topological polar surface area (TPSA), we tried to understand if the two groups could be discriminated within the chemical space based on their chemical properties. To better appreciate any existing difference between the two groups, we computed a principal component analysis (PCA). In brief, PCA is a multivariate technique that is used to reduce the dimension of the dataset while retaining as much information from the data as possible, alongside maximizing the variance of the data by defining principal components. The results are represented by score and loadings plots in Fig S2 A and S2 C. The PC score graph (Fig. S2 A) provides a visual representation of the dimension reduction achieved by PCA, while the PC loadings plot (Fig. S2 C) represent the correlation between variables and the principal components.

Based on parallel analysis which is a method for selecting principal components that accounts for variance in the data due to random error or noise (Fig. S3), four components have been selected accounting for $74 \%$ of the variance. With PC 1 and PC2 about $55 \%$ of the variance was explained and high and low permeable compounds can be discriminated on PC 1 which accounts for the highest variance. As expected, the loadings plot shows that permeability is negatively correlated with molecular descriptors associated with polarity (i.e., PSA, HBD, HBA), as they are arranged in a specular fashion with respect to the scores of the highly permeable group. Yet, a negative correlation is observed also with descriptors linked to molecular complexity (i.e., total molecular weight, non-H atoms, total surface area), and apparently no relation with lipophilicity (cLogP). This, for some extent, is somehow contradictory as numerous significant correlations between lipophilicity and drug passive permeation have been established.

The distribution of the tested drugs in the chemical space based on their behavior in the presence of mucus is challenging to interpret (Fig. S2 B). In the score plot, a general overlap of the three groups is observed, making it difficult to discriminate between each category. 


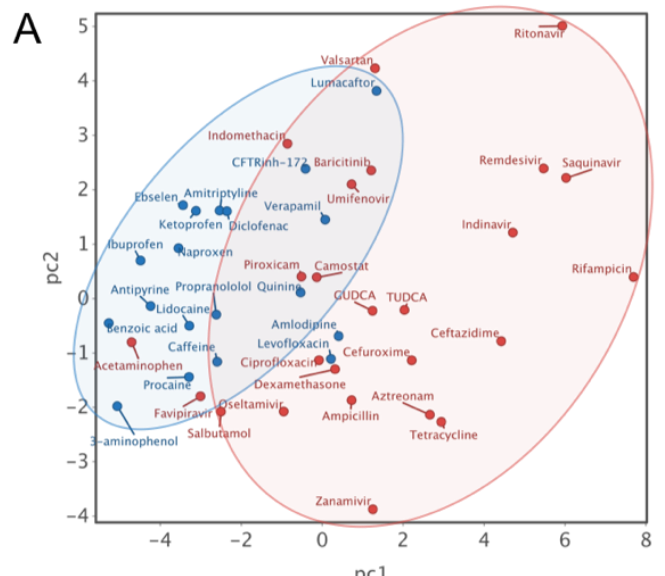

Papp classification binary (categorical) high $\bullet$ low

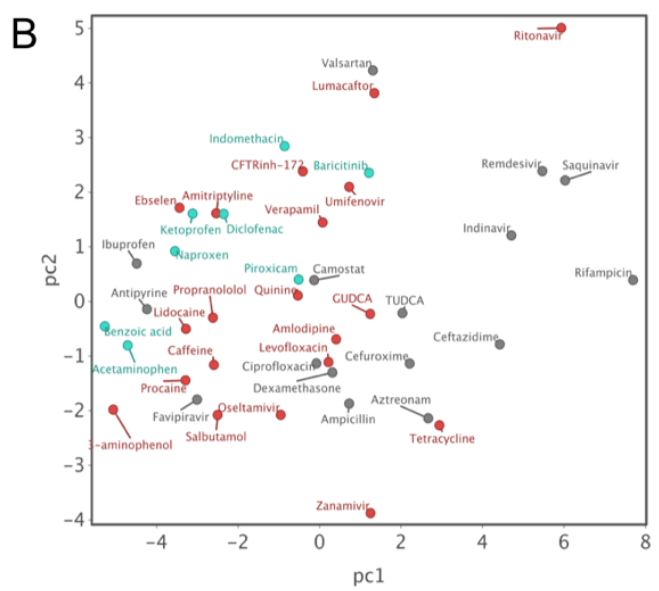

Effect of mucus on Papp (categorical) - Decreased O Increased ONone

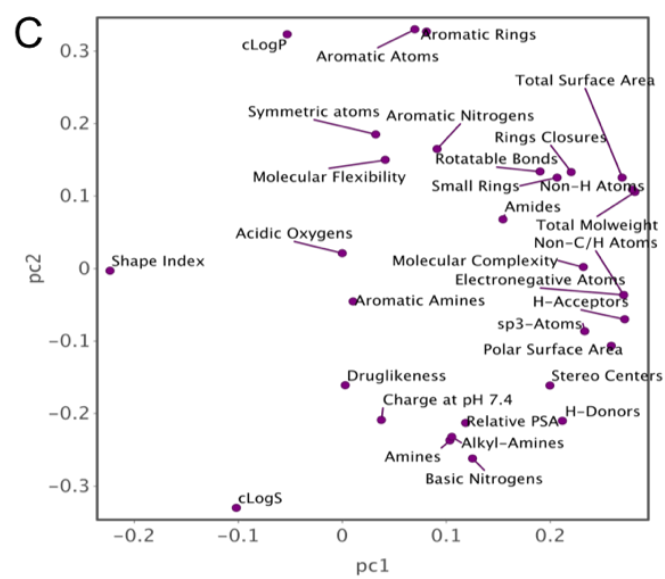

Figure S4. Principal component analysis of the dataset. (Fig S2 A) score plot colored based on the classification in high and low permeability. The blue and red circles define the discrimination of the two categories on the first principal component. (Fig S2 B) score plot colored by the effect of mucus over the permeability in the mucus-PAMPA system. Here we cannot see a clear discrimination on any of the principal 
components between the three categories. (Fig. S2 C) loading plot representing the correlation between the variables and the principal components.

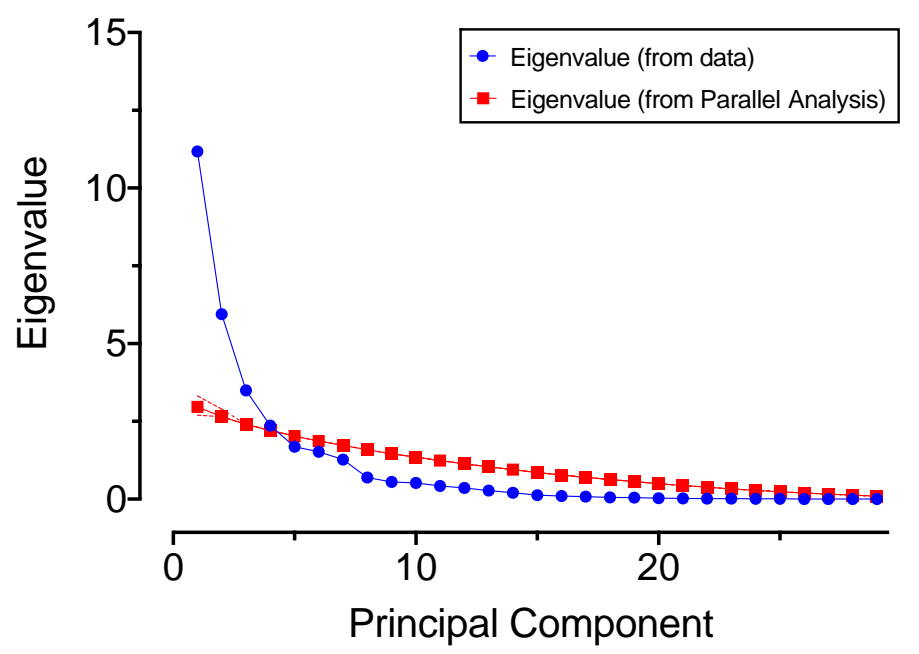

Figure S5. Selection of components based on parallel analysis. By retaining only those PCs with eigenvalues greater than the $95^{\text {th }}$ percentile of the simulated eigenvalues, it is ensured that the variance explained by these PCs likely represents "real" variance and not variance due to noise. 

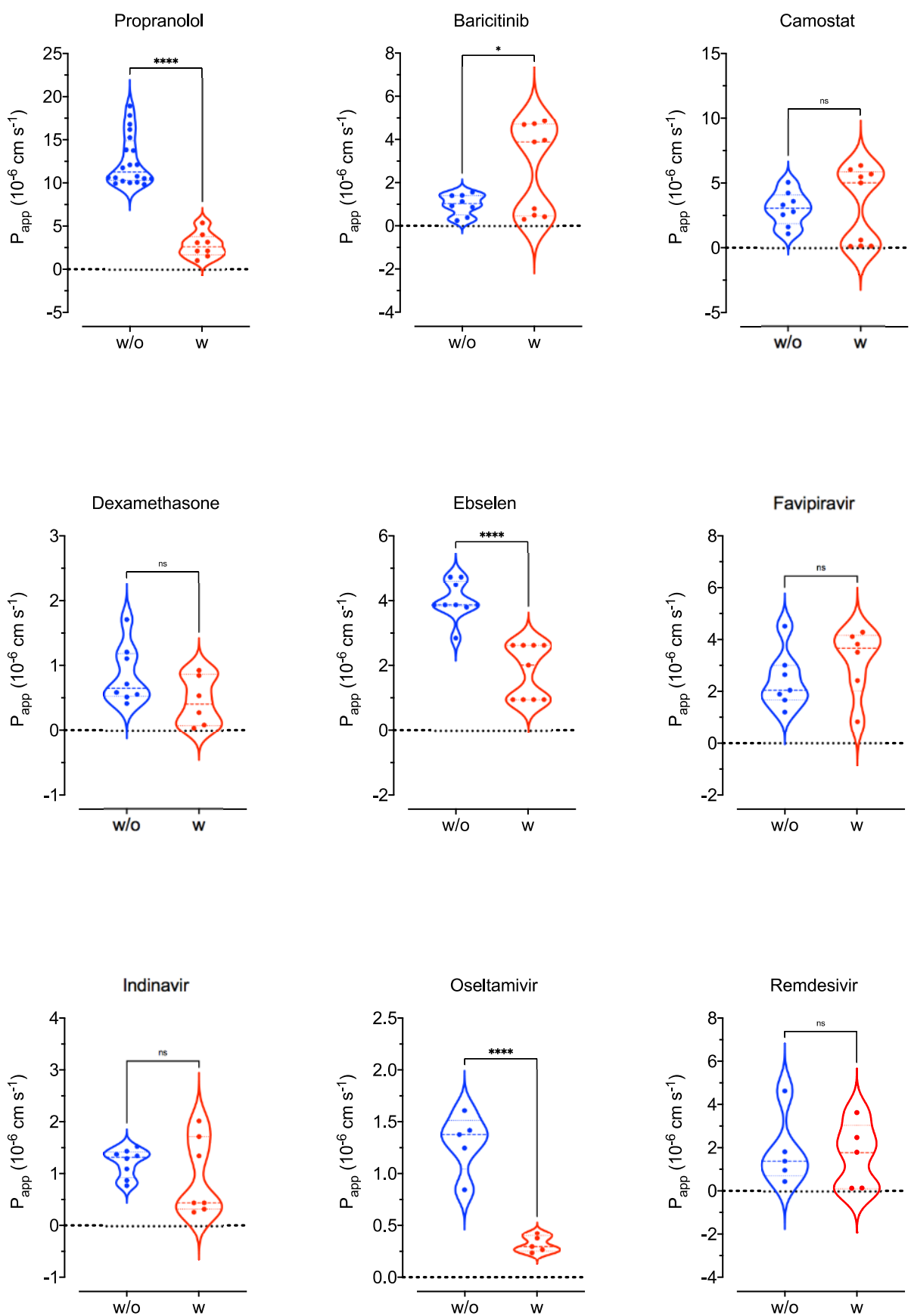

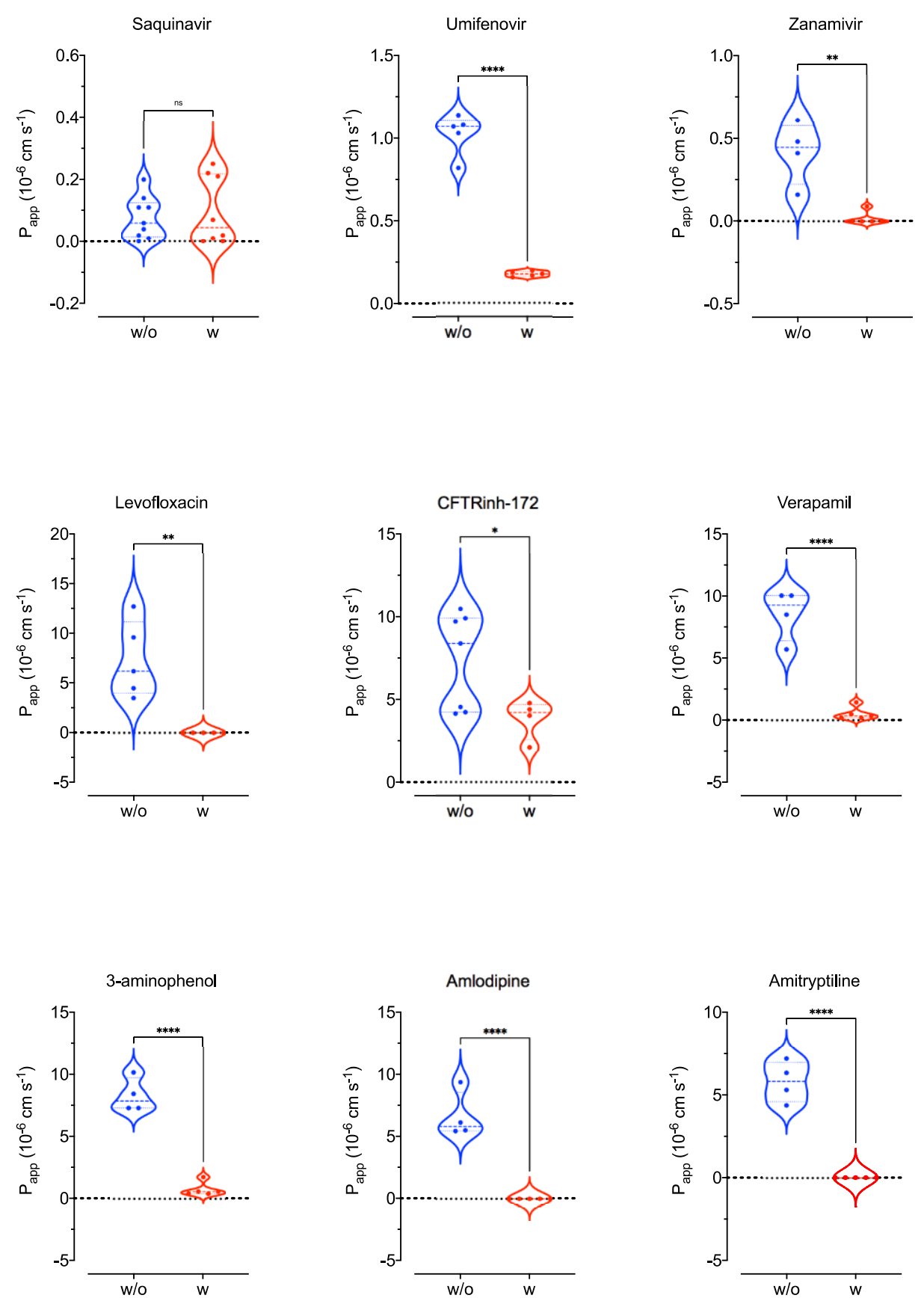

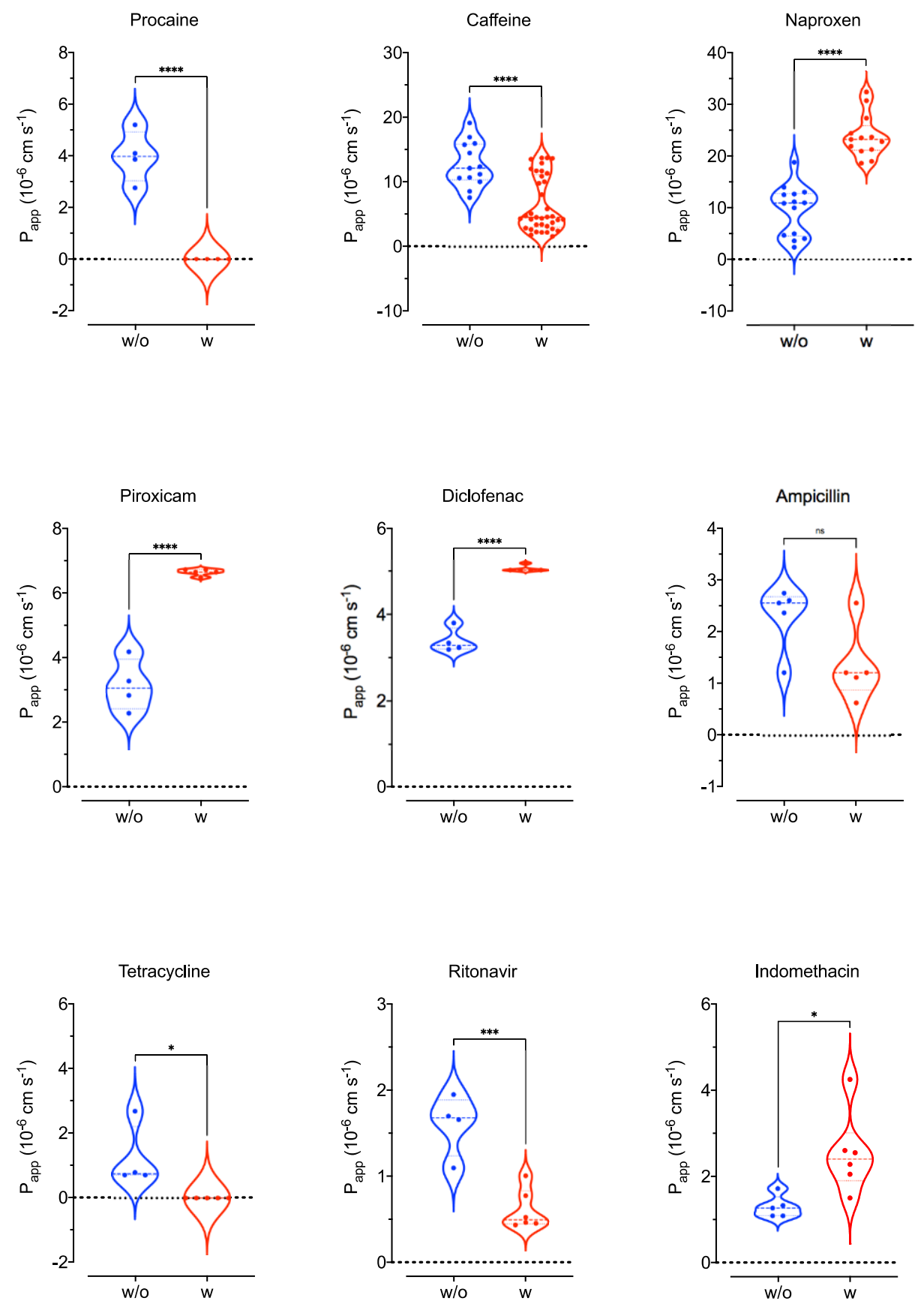

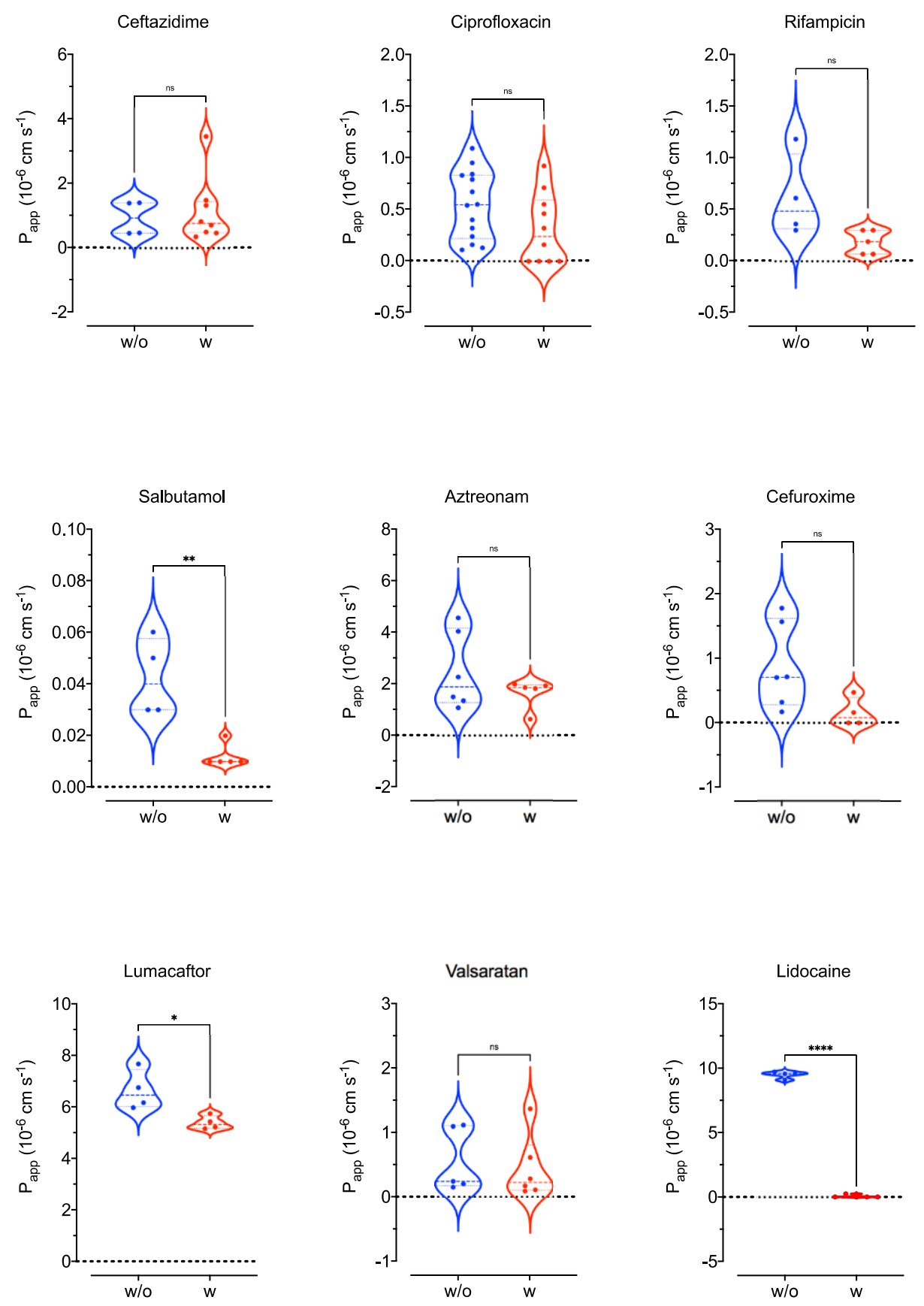

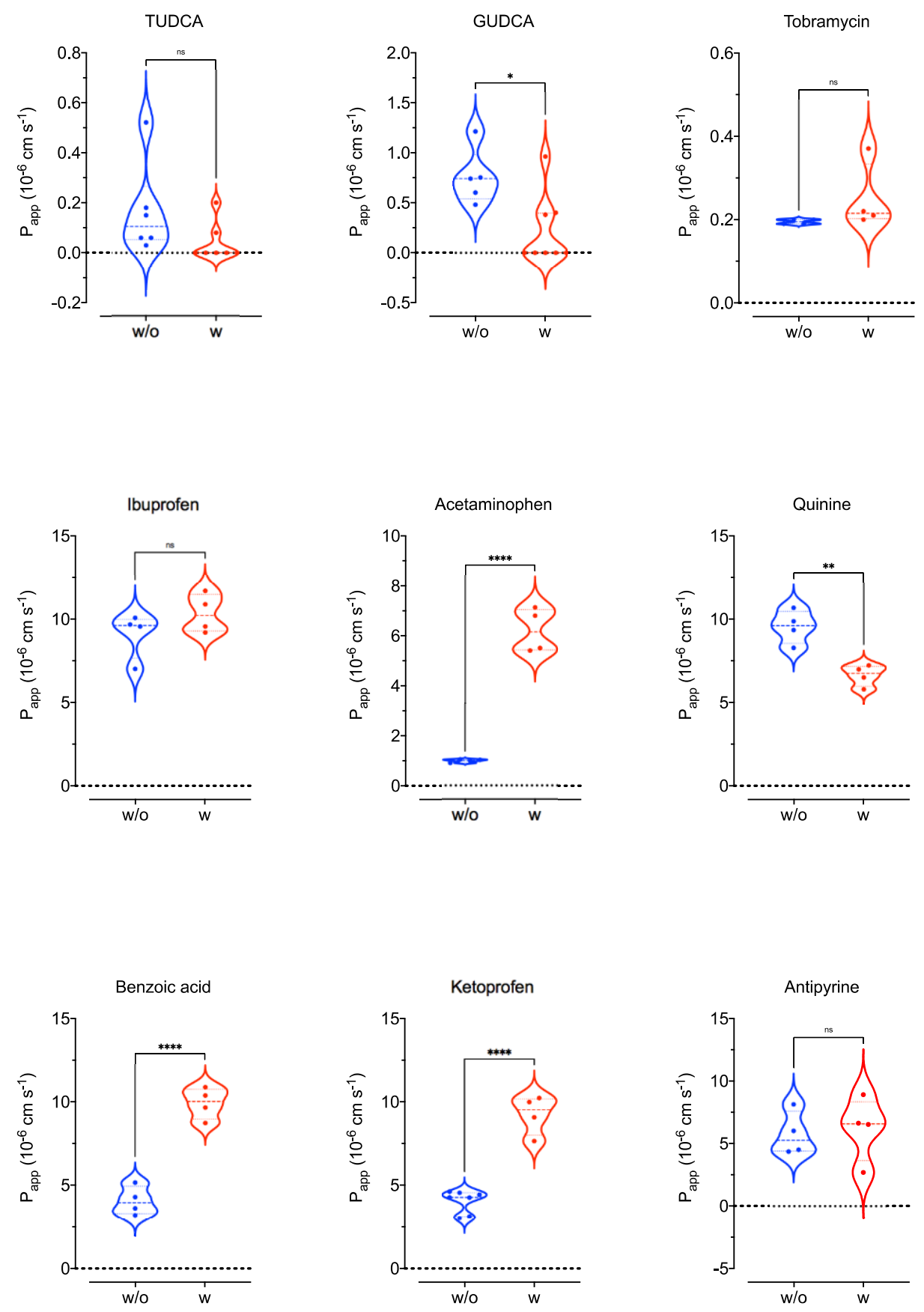

Figure S6. Comparison of the permeability recorded on PAMPA without (blue) and with mucus (red) for the tested compounds. 


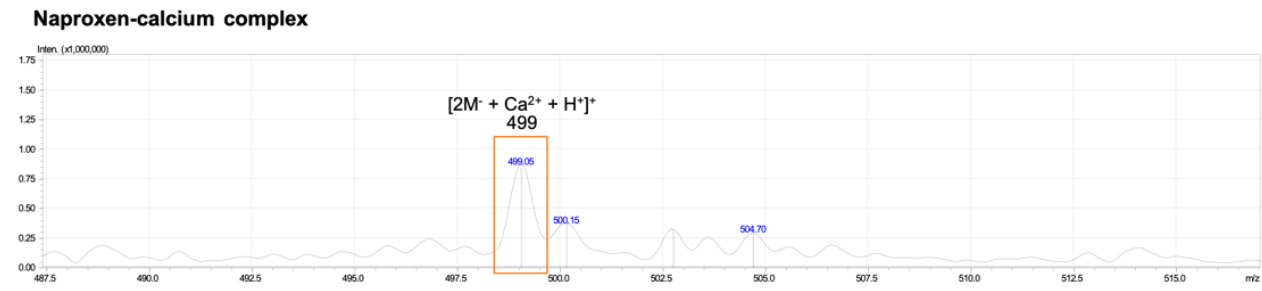

Figure. S7. The ESI-MS spectrum of the naproxen-calcium complex.

\section{References}

(1) Chen, X.; Murawski, A.; Patel, K.; Crespi, C. L.; Balimane, P. V. A Novel Design of Artificial Membrane for Improving the PAMPA Model. Pharm. Res. 2008, 25 (7), 1511-1520. https://doi.org/10.1007/s11095-007-9517-8.

(2) Kerns, E. H.; Di, L.; Petusky, S.; Farris, M.; Ley, R.; Jupp, P. Combined Application of Parallel Artificial Membrane Permeability Assay and Caco-2 Permeability Assays in Drug Discovery. J. Pharm. Sci. 2004, 93 (6), 1440-1453. https://doi.org/10.1002/jps.20075. 